\title{
Wearable devices for the detection of COVID-19
}

\author{
Wearable electronic devices, which allow physiological signals to be continuously monitored, can be used in the \\ early detection of asymptomatic and pre-symptomatic cases of COVID-19.
}

\section{H. Ceren Ates, Ali K. Yetisen, Firat Güder and Can Dincer}

N earable devices, such as activity trackers and smartwatches, can provide unique insights into our health and well-being ${ }^{1,2}$. Unlike conventional testing in a clinical setting, which may occur a few (or less) times a year, wearables offer continuous access to real-time physiological data. This allows deviations from a person's 'usual' baselines to be detected: an approach to healthcare that is fundamentally different from current practice, which predominantly compares physiological measurements to population statistics. And during the coronavirus disease 2019 (COVID-19) pandemic, the potential of wearable health devices has become increasingly apparent ${ }^{3-7}$.

To start, and writing in Nature Biomedical Engineering, Xiao Li, Michael Snyder and colleagues at the Stanford University School of Medicine and Case Western University have shown that smartwatch data - heart rate, number of daily steps and sleep time - can detect pre-symptomatic cases of COVID-198. The researchers analysed the data (collected with devices such as Fitbits and Apple watches) of 32 infected individuals, identified from a pool of over 5,000 participants, and explored correlations between different metrics in order to detect aberrant physiology. They looked, in particular, at elevated resting heart rates (relative to a previous healthy window), and increased heart rates relative to number of steps. Twenty-six of the COVID-19 positive participants produced aberrant physiological signals 4 to 7 days in advance of the onset of symptoms or diagnosis; 6 of the COVID-19 positive participants did not produce easily identifiable signals before the onset of symptoms. Thus, $81 \%$ of the participants could be identified as potentially COVID-19 positive. The researchers also created an online detection algorithm that could be used to identify the early stages of infection via real-time heart rate monitoring.

Elsewhere, and writing in Nature Medicine, Giorgio Quer and colleagues examined how similar smartwatch data plus self-reported symptoms can be used to detect COVID-199. The researchers - who are based at the Scripps Research

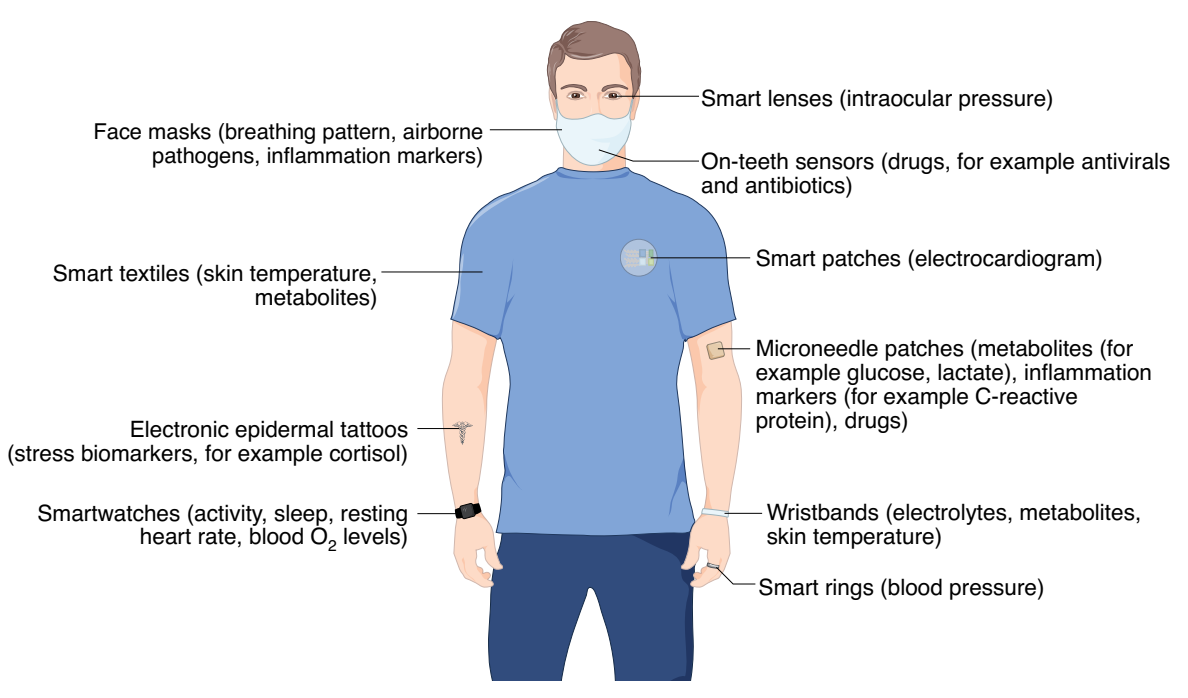

Fig. 1 | The future of wearable electronic devices. Currently, smartwatches provide information such as heart rate, sleep time and activity patterns. In the future, this could be augmented with new classes of wearable devices that monitor, for example, concentrations of cortisol for tracking stress (using electronic epidermal tattoos), biomarkers of inflammation and levels of blood $\mathrm{O}_{2}$ (microneedle patches), skin temperature (electronic textiles), blood pressure (smart rings), concentration of ions (wristbands), intraocular pressure (smart contact lenses), the presence of airborne pathogens and breathing anomalies (face masks), and the concentration of therapeutic drugs (on-teeth sensors) 2,10,12-16. Such emerging low-cost wearable sensing technologies, monitoring both physical parameters and biochemical markers, could be used to identify symptomatic and pre-symptomatic cases in future pandemics. The devices could also be used to remotely monitor the recovery of individuals undergoing treatment or self-isolating at home.

Translational Institute and CareEvolution in Michigan - enrolled over 30,000 participants for their study. Of these participants 3,811 reported at least one symptom, 54 (1.4\%) of which also reported testing positive for COVID-19 and 279 (7.3\%) testing negative. Resting heart rate data alone were not a significant discriminator between the positive and negative cases: the area under the curve (AUC) of the receiver operator characteristic (ROC) curve was 0.52 (an AUC of 0.50 corresponds to random classification between negative and positive cases, whereas 1 corresponds to perfect classification). But when resting heart rate, sleep and activity metrics were combined into a single metric, the AUC increased to 0.72 . And when sensor metrics were combined with self-reported symptoms, the AUC increased to 0.80 .

These studies do though have limitations. They are unable to differentiate COVID-19 from other viral infections, and they are predisposed to sample bias as older people and low-income populations would not typically own or have access to wearable devices. The detection methods also require large datasets to train the algorithms used, and thus for every new pathogen that yields different physiological and activity signatures, the (lengthy) studies would need to be repeated, delaying deployment. Furthermore, the lack of additional physiological information (the works depended only on heart rate) reduces diagnostic performance. 
The capabilities of these approaches could potentially be improved and extended with the use of emerging low-cost wearable sensors (Fig. 1). In particular, electronic sensors in the form of epidermal tattoos, contact lenses, textiles, face masks, wristbands and patches can help collect previously inaccessible physical and biochemical signals ${ }^{2,10-16}$. Inclusion of data from such additional sensors into the algorithmic decision-making process could reduce the effects of environmental, behavioural and other external factors (such as diet, travel, alcohol, stress, drug intake, other health conditions) on the diagnostic outcomes. By leveraging the known correlations between biomarkers and the physiological state of the body, this approach could substantially improve diagnostic performance, without requiring access to large training datasets.

The complexity of each wearable sensor depends on the analyte or physical quantity of interest and the goal of the measurement (relative versus absolute, qualitative versus quantitative). It is, of course, not practical to wear many different sensors at one time, but the functionality of a system can be extended by including different transducers (that is, multimodal devices) or by evaluating analytes simultaneously in a multiplexed fashion. Multimodal and/ or multiplexed measurements can also improve the performance of individual sensors by providing additional information for compensation and calibration, and creating more reliable signals. For example, concentrations of analytes in sweat are influenced by internal factors (such as the rate of sweat) and external factors (such as the temperature within the measurement environment). The accuracy of the measurement is, therefore, influenced by the precise information collected from the local environment. Next-generation wearable devices must also provide sufficient levels of reliability and robustness to be successfully integrated into daily life. For example, electronic contact lenses are kept in a delicate environment for extended periods of time. The devices must, therefore, be biocompatible, energy efficient, compliant and compact, without compromising performance.

The use of face masks with integrated sensors is one of the most promising strategies for identifying infected individuals. During the current pandemic, face masks have become ubiquitous and thus exploiting the platform for continuous health monitoring could be feasible. Low-cost face masks with integrated sensors can offer direct access to many important parameters via exhaled breath, including patterns and rates of respiration, biomarkers of inflammation and the potential detection of airborne pathogens ${ }^{13,14}$.

However, collecting physiological data from an individual is just half of the story. Population-based data and modelling may not be sufficient to make accurate predictions about infected individuals. The decision-making process should, ideally, be individualized, requiring the continuous collection - and processing of large volumes of data from each person. With continued reductions in the price of low-power silicon electronics, and increases in computational power, the processing of data locally - decentralizing and accelerating the decision-making process - should be possible. To improve central coordination, essential disease-relevant information may also be directly communicated (via wireless networks) to healthcare agencies overseeing the efforts for containment and treatment. Such an integrated approach does though create various additional challenges, including those related to data security, encryption, ethics and appropriate regulation ${ }^{2,17}$.

As wearable devices produce ever-larger datasets, reliance on data mining and machine-learning-based computational approaches will increase. Such analysis of the complex links between sensor data and the physiological status of patients is likely to yield previously unknown correlations that lead to more accurate predictions. The COVID-19 pandemic has highlighted the potential of wearable electronic devices in healthcare. With continued innovation and development, the next generation of wearable sensors - and their ability to continuously monitor both physical parameters and biochemical markers - could play a key role in fighting the next pandemic.

\section{H. Ceren Ates ${ }^{1,2}$, Ali K. Yetisen ${ }^{3}$ Firat Güder ${ }^{4 凶}$ and Can Dincer (DD 1,2凶 ${ }^{1}$ Freiburg Center for Interactive Materials and Bioinspired Technologies (FIT), University of Freiburg, Freiburg, Germany. ${ }^{2}$ Department of Microsystems Engineering (IMTEK), Laboratory for Sensors, University of Freiburg, Freiburg, Germany. ${ }^{3}$ Department of Chemical Engineering, Imperial College London, London, UK. ${ }^{4}$ Department of Bioengineering, Royal School of Mines, Imperial College London, London, UK.

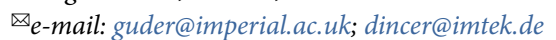

Published online: 25 January 2021

https://doi.org/10.1038/s41928-020-00533-1

References

1. Dunn, J., Runge, R. \& Snyder, M. Per. Med. 15, 429-448 (2018).

2. Yetisen, A. K., Martinez-Hurtado, J. L., Ünal, B., Khademhosseini, A. \& Butt, H. Adv. Mater. 30, 1706910 (2018).

3. Morales-Narváez, E. \& Dincer, C. Biosens. Bioelectron. 163, 112274 (2020).

4. Oran, D. P. \& Topol, E. J. Ann. Intern. Med. 173, 362-367 (2020).

5. Seshadri, D. R. et al. Front. Digit. Health 2, 8 (2020).

6. Li, X. et al. PLoS Biol. 15, e2001402 (2017).

7. Natarajan, A., Su, H.-W. \& Heneghan, C. npj Digit. Med. 3, 156 (2020).

8. Mishra, T. et al. Nat. Biomed. Eng. https://doi.org/10.1038/s41551 020-00640-6 (2020).

9. Quer, G. et al. Nat. Med. https://doi.org/10.1038/s41591-0201123-x (2020).

10. Dincer, C. et al. Adv. Mater. 31, 1806739 (2019).

11. Moreddu, R., Wolffsohn, J. S., Vigolo, D. \& Yetisen, A. K. Sens. Actuators B Chem. 317, 128183 (2020).

12. Ates, H. C. et al. Trends Biotechnol. 38, 1262-1277 (2020).

13. Güder, F. et al. Angew. Chem. Int. Ed. 55, 5727-5732 (2016).

14. Maier, D. et al. ACS Sensors 4, 2945-2951 (2019).

15. Gao, W. et al. Nature 529, 509-514 (2016).

16. Cotur, Y. et al. Adv. Funct. Mater. 30, 1910288 (2020).

17. Ferretti, L. et al. Science 368, eabb6936 (2020).

\section{Competing interests}

The authors declare no competing interests. 